PharmacoEconomics \& Outcomes News 854, p22 - 30 May 2020

\title{
Fremanezumab for migraine prevention: more effective, less costly
}

Fremanezumab provides better outcomes than erenumab as preventive treatment in episodic migraine for patients with inadequate response to prior preventive treatments and is less costly.

This is the main finding of a US study* that used a semi-Markov model with a 10-year time horizon to assess the cost effectiveness of fremanezumab compared to erenumab (140mg dosing) for the prevention of episodic migraine in adults who had responded inadequately to $2-4$ prior preventive treatments. ${ }^{* *}$ Costs included those associated with fremanezumab and erenumab acquisition as well as direct and indirect costs related to episodic migraine. The model was populated with clinical data from a network meta-analysis.

The model predicted that fremanezumab was less costly than erenumab, with an average incremental cost savings of \$US1795 per patient. Furthermore, fremanezumab was more effective, with incremental QALYs of 0.037 per patient and a reduction in 33.3 migraine days per 28 days per patient. When indirect costs were not included in the model, fremanezumab still dominated erenumab, with an average incremental cost savings of \$936.96 per patient.

* The study was to be presented at the 72nd Annual Meeting of the American Academy of Neurology, which was cancelled due to COVID-19 (originally scheduled 25 April to 1 May 2020 in Toronto, Canada).

** The work on these analyses was supported by Teva Pharmaceuticals.

Smolen L, et al. 10-Year Cost-effectiveness Analyses of Fremanezumab Compared to Erenumab as Preventive Treatment in Episodic Migraine for Patients With Inadequate Response to Prior Preventive Treatments. 72nd Annual Meeting of the American Academy of Neurology : abstr. N/A, 25 Apr 2020. 